\title{
Magnetic Global Monopoles from Torsion
}

\author{
Sarben Sarkar ${ }^{1, a}$ \\ ${ }^{1}$ Department of Physics, King's College London, U.K.
}

\begin{abstract}
In the search of avatars of new physics, we present a new classical solution for electromagnetic monopoles induced by global gravitational monopoles in the presence of a four-dimensional Kalb-Ramond axion field. The torsion induces the magnetic charge of the monopole.
\end{abstract}

\section{Introduction}

Electromagnetism is described by Maxwell's equations which are given by

$$
\begin{array}{ll}
\vec{\nabla} \cdot \vec{E}=\rho_{e} & \vec{\nabla} \cdot \vec{B}=0 \\
\vec{\nabla} \times \vec{E}+\frac{\partial \vec{B}}{\partial t}=0 & \vec{\nabla} \times \vec{B}-\frac{\partial \vec{E}}{\partial t}=\vec{j}_{e} .
\end{array}
$$

Electromagnetism is part of the the Standard Model (SM) of particle physics. In vacuum the charge density $\rho_{e}$ and current density $\vec{j}_{e}$ both vanish. The equations (1) have then an $O(2)$ duality symmetry

$$
\left(\begin{array}{c}
\vec{E} \\
\vec{B}
\end{array}\right) \rightarrow\left(\begin{array}{cc}
\cos \alpha & \sin \alpha \\
-\sin \alpha & \cos \alpha
\end{array}\right)\left(\begin{array}{c}
\vec{E} \\
\vec{B}
\end{array}\right) .
$$

If there is now a non-vanishing $\rho_{e}$, then the duality can be maintained if there is a monopole magnetic charge density $\rho_{m}: \vec{\nabla} \cdot \vec{B}=\rho_{m}$. The duality transformation is extended by requiring that

$$
\left(\begin{array}{c}
\rho_{e} \\
\rho_{m}
\end{array}\right) \rightarrow\left(\begin{array}{cc}
\cos \alpha & \sin \alpha \\
-\sin \alpha & \cos \alpha
\end{array}\right)\left(\begin{array}{c}
\rho_{e} \\
\rho_{m}
\end{array}\right) .
$$

Although no monopole has yet been found, the allure of duality symmetry between electric and magnetic fields and resulting monopoles [1] has resulted in the search for monopoles by the MoEDAL experiment [2] at CERN. This experiment, more generally, is devoted to the detection of highly ionising long-lived massive particles predicted by theories which go beyond the SM. A monopole could be one such particle but its mass range needs to be in the TeV range for it to have a chance of detection in current accelerator experiments. We shall give the salient points of some theories which predict the existence of monopoles in the next section. In the subsequent sections we will introduce a new model for monopoles which might provide an example of a detectable monopole.

\footnotetext{
ae-mail: sarben.sarkar@kcl.ac.uk
} 


\section{Magnetic Monopoles}

For a magnetic monopole at the origin (at $\vec{r}=0$ ) with charge $Q_{m}$, the $\vec{B}$ field has the form

$$
\vec{B}=\frac{Q_{m}}{4 \pi} \frac{\vec{r}}{r^{3}}
$$

where $r=|\vec{r}|$. Dirac [3] argued that the requirement of a Hilbert space structure for the quantum theory of an electrically charged particle of charge $e$ in the magnetic field of a monopole implied that $Q_{m}$ is quantised:

$$
Q_{m} e=2 n \pi
$$

for integer $n$. Hence, in terms of the fine structure constant $\alpha_{e}\left(\approx \frac{1}{137}\right)$

$$
\frac{Q_{m}}{e}=\frac{n}{2 \alpha_{e}}
$$

and so $Q_{m}$ is large compared to $e$.

\subsection{Monopoles in spontaneously broken gauge theories}

Grand unified theories, examples of models beyond the SM, allowed the exciting possibility that, in some circumstances, monopoles can exist. An example of a classical solution leading to a monopole was found in a gauge theory where the gauge symmetry was spontaneously broken by elementary scalar fields [4]. In this example the gauge group is $S U(2)$ which is spontaneously broken to $U(1)$ the gauge group of electromagnetism. Two of the three vector bosons in the theory acquire a mass $M_{v}$ and the mass of the monopole is approximately $\frac{4 \pi M_{v}}{e^{2}}$. This pattern is replicated in grand unified theories when a simple group is broken down to the group $U(1)$. Typically this leads to monopoles of mass determined by the scale of grand unified theories [5] and so the predicted monopoles have masses of order around $10^{17} \mathrm{GeV}$. It is hard to see within this framework how low mass monopoles can be found. Hence, although the models are very elegant, such monopoles cannot be found through accelerator searches. This fact has motivated us to seek a framework which moves away from the paradigm of spontaneously broken gauge symmetries.

\subsection{Monopoles from torsion}

We will construct a model where we replace the non-Abelian gauge bosons in models of monopoles in grand unified theories with the gravitational multiplet of string theory. The Kalb-Ramond field [6], which appears as part of the gravitational multiplet in string theory, will be denoted by torsion.The gravitational multiplet thus is comprised of the graviton, the dilaton and the torsion field. Our model, in addition to the gravitational multiplet, will require a triplet of strongly self-interacting scalar fields associated with the spontaneous symmetry breaking of a global $S U(2)$ group. The Lagrangian for our model [7] is

$$
\begin{aligned}
L & =(-g)^{1 / 2}\left\{\frac{1}{2} \partial_{\mu} \chi^{a} \partial^{\mu} \chi^{a}-\frac{\lambda}{4}\left(\chi^{a} \chi^{a}-\eta^{2}\right)^{2}-R\right. \\
& \left.+\frac{1}{2} \partial_{\mu} \Phi \partial^{\mu} \Phi-V(\Phi)-\frac{1}{12} e^{-2 \gamma \Phi} H_{\rho \mu \nu} H^{\varrho \mu \nu}-\frac{1}{4} e^{-\gamma \Phi} f_{\mu \nu} f^{\mu \nu}\right\}
\end{aligned}
$$

where $\chi^{a}, a=1,2,3$ form a scalar triplet of fields under $S U(2) ; g=\operatorname{det}\left(g_{\mu \nu}\right)$ is the determinant of the metric tensor $g_{\mu \nu} ; R$ is the Ricci scalar for $g_{\mu \nu}$; the antisymmetric tensor field strength $H_{\rho \mu \nu}$ for $B_{\mu \nu}$ 
the Kalb-Ramond field (torsion) is given by $H_{\rho \mu \nu}=\partial_{[\rho} B_{\mu v]} ; \Phi$ is the scalar dilaton field; $\gamma$ is a finite real parameter. The case $\gamma=1$ naturally arises in string theory effective actions and we shall restrict ourselves to this case. We can derive the following equations of motion:

$$
\begin{aligned}
g^{\nu \beta} \chi_{, \nu \beta}^{a}+\frac{1}{\sqrt{-g}} \partial_{\nu}\left(\sqrt{-g} g^{\nu \beta}\right) \chi_{, \beta}^{a}=-\lambda \eta^{2}\left(\chi^{b} \chi^{b}-\eta^{2}\right) \chi^{a}, \\
\nabla_{\kappa}\left(e^{-2 \gamma \Phi} H^{\kappa \beta \gamma}\right)=0, \\
\nabla_{\lambda}\left(e^{-\gamma \Phi} f^{\lambda \kappa}\right)=0,
\end{aligned}
$$

and

$$
G_{\mu v}=g_{N} \Theta_{\mu \nu}
$$

where $\Theta_{\mu \nu}$ is the energy-momentum tensor and, in terms of Newton's constant $G_{N}, g_{N}=8 \pi G_{N}$. These equations are supplemented with the Bianchi identity for the Kalb-Ramond field strength:

$$
\epsilon^{\mu \nu \lambda \rho} \partial_{\rho} H_{\mu \nu \lambda}=0
$$

Furthermore in 4-dimensions the Kalb-Ramond field strength is dual to a pseudoscalar ("axion"-like) field $b$. The remaining dilaton equation of motion is

$$
e^{2 \Phi} \partial_{\mu} b \partial^{\mu} b-\frac{1}{4} e^{-\Phi} f_{\mu \nu} f^{\mu \mu}-\frac{\delta V(\Phi)}{\delta \Phi}+O(\partial \Phi)=0
$$

where we did not write explicitly the terms involving $\partial_{\mu} \Phi$, since we will be interested in situations in which the dilaton is stabilised to a constant value $\Phi=\Phi_{0}$. (It is necessary to be aware of the units of variables and so we recast the equations in terms of dimensionless variables:

$$
W \rightarrow \frac{W}{\sqrt{g_{N}}}, \quad r \rightarrow \sqrt{g_{N}} r, \quad b \rightarrow \frac{b}{\sqrt{g_{N}}}, \quad \eta \rightarrow \frac{\eta}{\sqrt{g_{N}}} .
$$

The equations satisfied by these rescaled variables are the same as for the dimensional ones but with $g_{N}$ replaced by 1.)

\section{Classical Configurations for Monopoles from Torsion}

We will consider a class of static solutions of the equations (8), (9), (10), (11), (12) and (13) by making the ansätze [7]

$$
g_{\mu \nu}=\left(\begin{array}{cccc}
B(r) & & & \\
& -A(r) & & \\
& & -r^{2} & \\
& & & -r^{2} \sin ^{2} \theta
\end{array}\right)
$$

and

$$
f_{\mu \nu}=\left(\begin{array}{cccc}
0 & 0 & 0 & 0 \\
0 & 0 & 0 & 0 \\
0 & 0 & 0 & 2 r \sin \theta W(r) \\
0 & 0 & -2 r \sin \theta W(r) & 0
\end{array}\right)
$$


The associated magnetic field has only a radial component, which in contravariant form reads:

$$
\mathcal{B}^{r}=\epsilon^{r \theta \phi} f_{\theta \phi}=\frac{1}{\sqrt{-g}} \eta^{r \theta \phi} f_{\theta \phi}=\frac{2}{\sqrt{A B}} \frac{W(r)}{r},
$$

where $\eta^{r \theta \phi}=+1$, etc is the three-space totally antisymmetric symbol, and we took into account Eq. (15). The electric field is zero.

The ansatz for the scalar field is

$$
\chi^{a}=\eta f(r) \frac{x^{a}}{r}
$$

which is similar to the ansatz used in the 'Hooft-Polyakov monopole model (although other details of our model are quite different). The last remaining equation, derived from (12), is

$$
\frac{d}{d r}\left(\sqrt{\frac{B(r)}{A(r)}} r^{2} \frac{d b}{d r}\right)=0
$$

Its solution is

$$
b^{\prime}(r)=\frac{\varsigma}{r^{2}} \sqrt{\frac{A(r)}{B(r)}}
$$

where $\varsigma$ is a constant of integration which measures the strength of the Kalb-Ramond field strength.

\subsection{Asymptotic solutions}

Recently [7] an asymptotic analysis (for both large and small $r$ ) has been performed on the equations (8), (9), (10), (11), (12) and (13). The radial component $\mathcal{B}^{r}$ of the magnetic field is monopole-like and given by

$$
\mathcal{B}^{r}=\sqrt{2} \frac{1}{\sqrt{A B}} \frac{\varsigma}{r^{2}} .
$$

On ignoring deficit angles [8], the product $A(r) B(r)$ behaves asymptotically as:

$$
A(r) B(r)=1+\varepsilon(r)
$$

with $\varepsilon(r) \sim O\left(r^{2}\right)$ as $r \rightarrow 0$ and $\varepsilon(r) \sim O\left(r^{-1}\right)$ as $r \rightarrow \infty$. Moreover, as can be seen from (21),

$$
W(r) \sim \frac{\varsigma}{r}
$$

Also $B(r)$ has the Reissner-Nordström form:

$$
B(r)=1-\frac{2 M}{r}+\frac{2 \zeta^{2}}{r^{2}} .
$$

The asymptotics also suggest that $f(r)$ has a kink (or domain wall) shape which supports a bag-like structure of the monopole configuration: $f(r)$ is $O(r)$ for small $r$ and approaches 1 as $r \rightarrow \infty$. Work is in progress on finding a class of interpolating solution which connects smoothly the small and large $r$ behaviour discussed above. 


\subsection{Monopole energy}

To make an estimate of the monopole mass, we shall use the analytic form of the solution in the two asymptotic regimes of small and large (radial) distances from the monopole centre. The monopole mass is concentrated in the core region whose size we will estimate following arguments similar to those in ref. [8]. The total energy (i.e. (rest) mass $\mathcal{M}$ ) is given by the integral over three space of the time-time component of the stress energy tensor:

$$
\mathcal{M}=\int \sqrt{-g} d^{3} x\left[\frac{2 W^{2}}{B r^{2}}+\frac{\left(b^{\prime}\right)^{2}}{4 B A}+\eta^{2}\left(\frac{f^{2}}{B r^{2}}+\frac{\left(f^{\prime}\right)^{2}}{2 B A}\right)+\frac{\lambda \eta^{4}}{4 B}\left(f^{2}-1\right)^{2}\right] .
$$

This equation needs to be regularised because the third term in the square brackets of the integrand leads to a linear divergence due to its large $r$ behaviour. We are currently seeking a natural procedure that leads to a bag-like structure and an effective cut-off in the integral. In [7] the size of the core was phenomenologically taken to be

$$
L=\xi \lambda^{-1 / 2} \eta^{-1}
$$

with $\xi$ a parameter. Using such a spatial infrared cutoff $L$, we estimate the mass of the monopole to be

$$
\mathcal{M} \sim 4 \pi \frac{\eta^{2}}{1-\eta^{2}} \int_{0}^{L} d r \sim 4 \pi \eta^{2} L,
$$

for $\eta \ll 1$ (or in terms of the dimensionful quantities $\eta \ll M_{\mathrm{Pl}}$, where $M_{\mathrm{Pl}}$ is the reduced Planck mass); this estimate is consistent phenomenologically (see below). Physically, and following the logic of ref. [8], which discusses self-gravitating global monopoles in the absence of both electromagnetic fields and (Kalb-Ramond) torsion, we may assume that the mass of the monopole is concentrated in its core, whose size is $L$, and outside this the scalar field configuration approaches its constant vacuum expectation value, that is $f \sim 1$. In [7] $\xi$ was rewritten as $\xi=\frac{3}{2} \sqrt{\frac{\lambda}{\alpha}}$, with $0<\alpha<1$ which can be arranged to be of $\mathrm{O}(10)$. These considerations lead to an estimate [7] of the monopole mass

$$
\mathcal{M} \sim 12 \pi \sqrt{\frac{1}{\alpha}}(1-\alpha)|\varsigma| \eta,>0 .
$$

The important point to notice is that the mass is proportional to the Kalb-Ramond field strength ("torsion charge") $\varsigma$ and independent of $\lambda$ (in leading order for large $\lambda$ ). We hope to clarify this point in our ongoing work on interpolating solutions.

\section{Constraining the model by experiment}

We have outlined how Kalb-Ramond axion fields could generate a magnetic monopole. There is spontaneous symmetry breaking of a global internal symmetry which is essential for producing a self-gravitating global monopole with a deficit angle. In the limit $\lambda \rightarrow \infty$ the scalar fields have no propagating degrees of freedom and are confined to classical values that interpolate between the two expectation values that minimize the potential, that is between zero and $\eta$. Without non-zero KalbRamond field strengths ("torsion"), we showed that no magnetic monopole charge is induced by the global monopole (in the limit of large $\lambda$ but there are $O(1 / \lambda)$ contributions of course).

The existence of the magnetic charge implies high ionization, while the smallness of the monopole mass (as compared to the Planck scale) makes our magnetic monopole model falsifiable in the current round of the LHC [9]. The scalars in the model do not represent the SM Higgs, but elementary 
defects (or composites of heavy fermions) associated with a spontaneous breaking of $\mathrm{O}(3)$ symmetry; the phenomenological parameters $\lambda$ and the vacuum expectation value $\eta$, as well as the core size parameter $\xi$ can be constrained by experiments, if one accepts the loose definition of the core (26) ; the core dimension is some large distance (compared to the Planck length) such that, for distances larger than this, the solution for the scalar field configuration is $f \simeq 1$.

Aside from uncertainties in the current estimate of the core, a test of our model and experiment has to address the following:

- For large $\lambda$ losses due to production of Goldstone bosons need to be estimated for monopoleantimonopole pairs;

- We need to develop an understanding of the production of global monopoles from the collision of SM particles;

- The bounds from LHC experiments [9] have been obtained from perturbative Drell-Yan processes from the decay of virtual photons into monopole-antimonopole pairs and so are not reliable for our monopoles;

- In an effective field theory framework [10], the derivative of the Kalb-Ramond axion field $\partial_{\mu} b$ couples to the axial fermion current $\psi_{i} \gamma^{\mu} \gamma^{5} \psi_{i}$, where $i$ runs over fermion species of the SM, but the scalars couple only gravitationally to the SM fields (unless they are composites of heavy fermions which may couple to SM fields through loops). Hence the actual production mechanism of the global monopole solution at colliders, such as the LHC, and their detection, needs to be examined carefully. We stress that the monopole production is certainly expected to be strongly suppressed unless $\lambda$, as assumed by us, is large.

\section{References}

[1] E. Corrigan, D. I. Olive, D. B. Fairlie and J. Nuyts, Nucl. Phys. B 106, 475 (1976). P. Goddard and D. I. Olive, Rept. Prog. Phys. 41, 1357 (1978). see also: Y. M. Shnir, Magnetic monopoles, Berlin, Germany: Springer (2005) 532 p, in particular section 5.1.4.

[2] M. Fairbairn and J. L. Pinfold, Contemp. Phys. 58 (2017) no.1, 1.

[3] P. A. M. Dirac, Phys. Rev. 74, 817 (1948). Int. J. Theor. Phys. 17, 235 (1978).

[4] G. 't Hooft, Nucl. Phys. B 79, 276 (1974). A. M. Polyakov, JETP Lett. 20, 194 (1974) [Pisma Zh. Eksp. Teor. Fiz. 20, 430 (1974)].

[5] H. Georgi and S. L. Glashow, Phys. Rev. Lett. 32, 438 (1974).

[6] D. J. Gross and J. H. Sloan, Nucl. Phys. B 291, 41 (1987). R. R. Metsaev and A. A. Tseytlin, Nucl. Phys. B 293, 385 (1987). See also: R. T. Hammond, Rept. Prog. Phys. 65, 599 (2002).

[7] N. E. Mavromatos and S. Sarkar, Phys. Rev. D 95 (2017) no.10, 104025 [arXiv:1607.01315 [hepth]].

[8] M. Barriola and A. Vilenkin, Phys. Rev. Lett. 63, 341 (1989).

[9] G. Aad et al. [ATLAS Collaboration], Phys. Rev. D 93, no. 5, 052009 (2016) [arXiv:1509.08059 [hep-ex]]; B. Acharya et al. [MoEDAL Collaboration], arXiv:1604.06645 [hep-ex].

[10] M. J. Duncan, N. Kaloper and K. A. Olive, Nucl. Phys. B 387, 215 (1992). M. de Cesare, N. E. Mavromatos and S. Sarkar, Eur. Phys. J. C 75, no. 10, 514 (2015) [arXiv:1412.7077 [hep$\mathrm{ph}]$. 